\title{
The onset of miliary tuberculosis presenting after an episode of angina, palpitations and cardiac stent placement
}

\author{
Suha Moten ${ }^{1 *}$, Alyssa Panganiban ${ }^{1}$, Anis Abdul Rauf ${ }^{2}$ and Jaqueline Walker ${ }^{3}$ \\ ${ }^{1}$ Loyola University, Chicago, IL, USA \\ ${ }^{2}$ Nephrologist and Critical Care Physician, Nephrology Associates of Northern Illinois Hinsdale, 911 N Elm St \#102, Hinsdale, IL 60521, USA \\ ${ }^{3}$ Department of Family Practice, AMITA Health Medical Group, Westchester, IL 60154
}

\begin{abstract}
The novel Coronavirus of 2019 (COVID-19) has dramatically reminded the world of the importance of noting pertinent travel history. Increasing global travel and immigration require physicians to include travel history when considering their differential diagnoses. In this report, we present a 75 -year-old male diagnosed with Miliary Tuberculosis (TB) after returning from vacation in Mexico. During his vacation, he had symptoms attributed to unstable angina and underwent a cardiac catheterization requiring the placement of a drug eluting stent. This case report discusses angina as a possible presenting symptom of Miliary TB. Cardiac arrhythmia is a known risk of TB and it should be suspected in patients with arrhythmias with a recent travel history, as it is estimated that $1 \%$ of all cases of TB have cardiac involvement. Ultimately, with the aging of the population and our increasing mobile population, Miliary TB must be considered more frequently in our differentials in the developed world due to the aging of the population and increased ease of international travel.
\end{abstract}

\section{Introduction}

Miliary Tuberculosis (TB) is a form of Tuberculosis which results from the hematogenous dissemination of the airborne bacteria, Mycobacterium tuberculosis. Miliary TB may arise from an acute infection or a latent infection and can be classified as pulmonary or extrapulmonary [2-4]. Infection occurs via inhalation of droplets containing bacilli $\mathrm{M}$. tuberculosis that reach the alveoli of the lungs. TB typically affects the lungs, liver, and bone marrow but may affect other organs, including brain tissue, meninges, or the pericardium. Once the tubercle bacilli are ingested by alveolar macrophages, they are either destroyed, inhibited, or they may multiply intracellularly. If the bacilli multiply, they may then spread to other organs and systems via lymphatic channels or the bloodstream $[4,5]$. Although $\mathrm{TB}$ is a common disease with a rising incidence even in the developed world, there is still much that is unknown about the less common form known as Miliary TB, including its varied presentation and its complex treatments. Miliary TB remains a diagnostic challenge, as it accounts for less than $2 \%$ of all confirmed cases of TB and up to $20 \%$ of all extrapulmonary TB (EPTB) cases in various clinical studies [6]. Miliary TB accounts for approximately $3 \%$ of incidence cases in the United States with an increased representation among young children, the elderly, or those with compromised immune function [7, 8]. Historically, Miliary TB has been a concern in children due to their relatively immature immune system. However, increases in the incidence of TB have mirrored the aging of the US population, as $80-90 \%$ of the cases of Tuberculosis occur in patients over the age of $50[9,10]$. Over the last three decades, Miliary TB is becoming more recognized among older adults either due to the reactivation of latent disease or new exposure. Because impaired immunity develops with age, the likelihood of new exposure to Miliary TB is higher for older adults [4, $6,11]$. The symptoms of Miliary TB may include, but are not limited to dyspnea, fever, fatigue, body soreness, gastrointestinal discomfort, and weight loss. These symptoms vary depending on the organs that are affected, and TB can mimic many other conditions [3, 12]. Mortality from Miliary TB has remained high at $25-30 \%$, and approaches $50 \%$ in some studies, despite the availability of effective treatment and due in part to the challenges encountered in making the diagnosis $[1,13]$. Since Dr. Robert Koch announced the discovery of the $m$. tuberculosis bacterium in 1882, communicable diseases, including the recent novel coronavirus outbreak in 2020 , have remained a significant challenge for physicians and public health.

The diverse symptoms associated with Miliary TB and the challenges associated with culturing mycobacteria can make diagnosis difficult and a high index of suspicion is required. For instance, patients that have TB of the kidney and urinary tract typically present with urinary symptoms similar to a "conventional bacterial cystitis." Only one third of patients with renal involvement present with an abnormal chest x-ray [14]. In patients with Miliary TB, many lifethreatening cardiac complications have been confidently identified [6]. Unstable angina may arise in patients with Miliary TB. Pathogens of cardiovascular disease are intracellular organisms that can introduce the human body to chronic or latent infection, causing inflammation, and thus can lead to atherosclerotic plaque formation. Because a latent Tuberculosis infection (LTBI) is associated with chronic inflammation, there is a link between cardiovascular events and TB [15]. On the other hand, pulmonary or extrapulmonary TB can increase the risk of a patient experiencing an acute myocardial infarction (AMI) and

${ }^{*}$ Correspondence to: Suha Moten, Loyola University, Chicago, IL, USA, Email: suhaimoten@gmail.com

Key words: tuberculosis, miliary tuberculosis, disease, latent TB, cardiac stent, angina, novel coronavirus, travel

Received: March 27, 2020; Accepted: April 14, 2020; Published: April 24, 2020 
unstable angina $[7,15]$. As a result, unstable angina can leave a patient more susceptible to contracting Miliary TB. In this report, we present a 75-year-old Caucasian male, who resided in Mexico in the late 1970s where he was exposed to tuberculosis and may have developed class 1 TB. This same TB may have been reactivated recently during his recent vacation to Mexico, and initially manifested with cardiac arrhythmia and angina due to his underlying cardiac disease. Upon his arrival back to the United States, his symptoms lead to his hospitalization and the lesions found on his $\mathrm{CT}$ of the abdomen and pelvis were suspicious for and ultimately proven to be Class 5 Miliary TB.

\section{Case report}

During his vacation in Mexico, the 75-year-old Caucasian gentlemen developed palpitations and a feeling of impending doom. He was seen in the emergency department in Puerto Vallarta, Mexico, where he was diagnosed with unstable angina due to abnormal stress test and had cardiac catheterization, revealing in stent stenosis of 2 previously placed stents and LAD stenosis. The previous year he had stents placed in Mexico that were non drug eluting due to low platelets. However, given the rapid restenosis, decision was made to place drug eluting stents for this event.

Since arriving back from Mexico, the patient had very poor oral intake and slept 18 to 20 hours per day. He presented to an Illinois hospital with abdominal pain, profound weakness, and an episode of hematochezia. He lost about 8 to 10 pounds in the weeks prior to admission, mainly due to postprandial discomfort. He attributed his abdominal pain to the same "stomach bug" that affected others and his wife at their condo in Mexico. The patient denied any personal history of renal disease, kidney stones, Crohn's disease, sprue, or any other gastrointestinal disorders. However, he does have a familial history of hyperlipidemia, Coronary Artery Disease from his paternal side, and ovarian, and rectal cancer from his maternal side. He has a history of heart disease, familial hyperlipidemia with statin intolerance, peripheral vascular disease, and mild hyponatremia. In the past, $\mathrm{He}$ had an extensive workup for his hyponatremia, including an evaluation for adrenal insufficiency, which was negative.

With Miliary Tuberculosis on the differential diagnoses list, he was questioned in detail on his lifestyle habits. He denied any illicit drugs, tobacco, abdominal surgeries, ethylene glycol consumption, NSAID use, or frequent exposure to contrast media. He admitted to consuming 1-2 alcoholic beverages per week. He was screened for HIV 1/2 antigen/antibody, which was negative. He also reported that he resided in Mexico for about 4 years in the 1970's and reported the history of positive PPD in late 1970's upon return to Chicago, IL. He had a negative chest $\mathrm{x}$-ray and received no treatment, as that was the standard of care at the time. Upon routine surveillance, his repeated PPD tests have been negative.

His hospitalization was complicated by multiple episodes of tachycardia with heart rates in the 150's with hypotension found to be supraventricular tachycardia which was managed with increased beta blockade. After positive stool cultures for cryptosporidium and giardia, negative QuantiFERON gold, and multiple biopsies including ileum on colonoscopy, paracentesis, bone marrow, laparoscopic peritoneal and omental biopsy, bronchoscopy while on a ventilator, and an open lung biopsy all with negative acid-fast stains, he was started on antitubercular treatment. He ultimately underwent a cardiac ablation post hospitalization.

He responded well to treatment overall. His condition is gradually improving and stabilizing. The first cultures grew acid fast bacilli 24 days after hospital presentation; which were ultimately found 8 weeks after admission to be mycobacterium tuberculosis without drug resistance. He was on treatment for a total of one year and is alive and well three years later.

\section{Discussion}

In most cases, symptoms and etiologies for Miliary TB are easily identifiable, however considering his age, concomitant infections and underlying cardiovascular disease may have led to $\mathrm{TB}$ reactivation and the progression of his underlying renal and cardiac dysfunction. In this case, the probable reactivation of dormant TB is suspected rather than a de novo case. Miliary Tuberculosis is usually treated with antibiotics for six to nine months. The current ten anti-TB agents approved by the U.S. Food and Drug Administration are dominantly INH RIF drugs [16]. However, Drug Resistant TB (DR-TB) that is resistant to at least one first line anti-TB drug is becoming more prevalent. DR-TB results when TB is inadequately treated, which results in the bacteria developing resistance to the drugs used; it usually triggered by noncompliance with treatment plan or exposure to a patient with DR-TB.

The differential diagnosis for our patient includes primarily infection (mycobacteria, Yersinia, and others) and Crohn's disease. It is unclear exactly why he had a reactivation of his tuberculosis at this particular time, but exposure to various viral illnesses and cryptosporidium during the most recent trip to Mexico may have resulted in a suppression of his immune system, allowing the tubercular bacteria to become active. Individuals with a greater frequency or duration of exposure to any stimulus have a higher risk for transmission and manifestation of TB. His kidney injury may have been a direct result of the small amount abdominopelvic ascites with superimposed mesenteric edema and omental nodularity with mild mesenteric lymphadenopathy which was suspicious for peritoneal metastatic disease [17] (Figure 1). The peritoneal enhancement on the $\mathrm{CT}$ at presentation was concerning for metastatic disease especially with concomitant blood in urine and darkening stool samples (Figure 2). As a result, impaired renal function affected the heart and the TB caused cardiac inflammation which contributed to his antecedent tachycardia and angina [18]. Hence, miliary TB needs to make it onto the differential diagnosis even

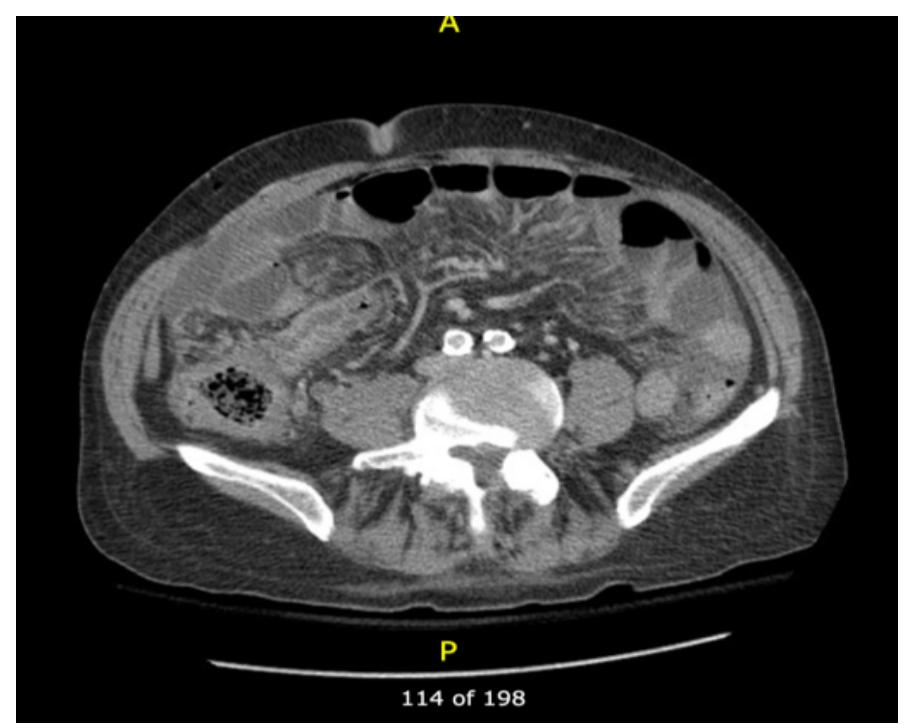

Figure 1. Wall thickening of the terminal ileum with adjacent lymphadenopathy in Abdomen and Pelvis CT (with contrast) 


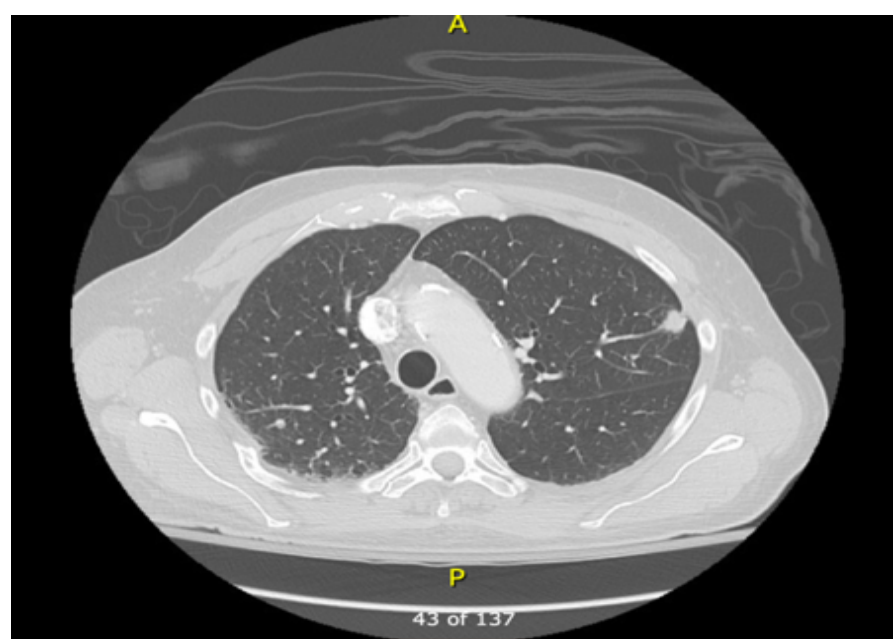

Figure 2. Opacity in both lungs in chest CT (with contrast)

in developed countries as it may be treatable if diagnosed. However, it shows to be fatal if the diagnosis or treatment is prolonged. TB is more common in developing nations than it is in the United States. However, the aging population in the United States and the increasing prevalence of travel makes tuberculosis a consideration even in the US. Air travel itself carries a relatively high risk of infection with TB [18].

Thus, travelers should avoid contact or prolonged time with known TB patients in enclosed environments such as clinics or hospitals. If a traveler suspects that they have been exposed to tuberculosis, then medical evaluation is indicated, and the traveler should make sure the physician knows the extent of travel. In the post-COVID era, protocol for travelers who anticipate possible exposure to infected individuals may need to be modified to call for a TB skin test, TB blood test, or COVID-19 swab test $[19,20]$. Due to the possibility of an initial false negative in both tests, especially in older patients, it is currently recommended to repeat a TB test 8 to 10 weeks after returning to the United States (two-step test). The recommendations for testing in this new era will evolve [21-23]. Our increasingly shrinking world with fluid borders means that physicians must keep highly infectious diseases and its many manifestations on their differential diagnoses lists in order to not miss these potentially fatal conditions. Nothing has killed more vulnerable human beings than infectious disease .

\section{Acknowledgement}

None to declare

\section{Financial disclosure}

None to declare

\section{Conflict of interest}

All authors declare no conflict of interest.

\section{Informed consent}

The patient has given their informed consent for the publication of this report.

\section{Author contributions}

SM was responsible for the data collection and writing the manuscript. AP was responsible for helping write the manuscript. AAR and JW were responsible for the patient's care and review of literature.

\section{References}

1. Khurana R, Shalhoub J, Verma A, Assomull R, Prasad SK, et al. (2008) Tubercular myocarditis presenting with ventricular tachycardia. Nat Rev Cardiol 5: 169-174. [Crossref]

2. Bernardo J, Reyn CFV, Baron EL (2020) Epidemiology and pathology of miliary and extrapulmonary tuberculosis.

3. Tuberculosis (2019) Mayo Clinic. Available at: https://www.mayoclinic.org/ diseasesconditions/tuberculosis/symptoms-causes/syc-20351250

4. Yoshikawa TT (1992) Tuberculosis in aging adults. J Am Geriatr Soc 40: 178-187. [Crossref]

5. American Thoracic Society and CDC (2000) Diagnostic standards and classification of tuberculosis in adults and children. Am J Respir Crit Care Med 161: 1376-1395. [Crossref]

6. Sharma SK, Mohan A, Sharma A (2012) Challenges in the Diagnosis \& Treatment of Miliary Tuberculosis. Indian J Med Res 135: 703-730. [Crossref]

7. Horsburgh RC, Reyn CFV, Baron EL (2020) Epidemiology of Tuberculosis in the Era of HIV.

8. Debi U, Ravisankar V, Prasad KK, Sinha SK, Sharma AK (2014) Abdominal tuberculosis of the gastrointestinal tract: revisited. World J Gastroenterol 20: 1483114840. [Crossref]

9. Rajagopalan S (2001) Tuberculosis and Aging: A Global Health Problem, Clin Infect Dis 33: 1034-1039. [Crossref]

10. Lipsitch M, Swerdlow DL, Finelli L (2020) "Defining the Epidemiology of Covid-19 — Studies Needed. "N Engl J Med 382: 1194-1196. [Crossref]

11. MacKay AD, Cole RB (1984) The problems of tuberculosis in the elderly. $Q J$ Med 53: 497-510. [Crossref]

12. Bobrowitz ID (1982) Active tuberculosis undiagnosed until autopsy. Am J Med 72 650-658. [Crossref]

13. Paules CI, Marston HD, Fauci AS (2020) Coronavirus Infections-More Than Just the Common Cold. JAMA. [Crossref]

14. Eastwood JB, Catherine M, Grange JM (2001) "Tuberculosis and the Kidney.” JASN 12: $1307-1314$.

15. Huaman MA, Henson D, Ticona E, Sterling TR, Garvy BA (2015) Tuberculosis and cardiovascular disease: linking the epidemics. Trop Dis Travel Med Vaccines 1: 10.

16. Adverse Events, Center for Disease Control and Prevention, 2016. Available from www.cdc.gov/tb/topic/treatment/adverseevents.htm

17. Healthline. Available from: https://www.healthline.com/health/Miliary-tuberculosis

18. "Can Flying Weaken Your Immune System? 30 Seconds to Fly Inc. Available from: www.30secondstofly.com/corporate-traveler/can-flying-weaken-your-immunesystem/.

19. Tierney D, Nardel EA (2018) "Miliary Tuberculosis (TB) - Infections, MSD Manuals

20. McIntosh K (2019) “Coronavirus Disease 2019 (COVID-19): Epidemiology, Virology, Clinical Features, Diagnosis, and Prevention.

21. Hoehl S, Rabenau H, Berger A, Kortenbusch M, Cinatl J, et al. (2020) "Evidence of SARS-CoV-2 Infection in Returning Travelers from Wuhan, China." $N$ Engl J Med 382: 1278-1280. [Crossref]

22. Underwood J, Cresswell F, Salam AP, Keeley AJ, Cleland C et al. (2017) Complications of miliary tuberculosis: low mortality and predictive biomarkers from a UK cohort. BMC Infect Dis 17: 295. [Crossref]

23. Dowdle WR (1989) A strategic plan for the elimination of tuberculosis from the United States. MMWR 21: 1-25. [Crossref]

Copyright: (C2020 Moten S. This is an open-access article distributed under the terms of the Creative Commons Attribution License, which permits unrestricted use, distribution, and reproduction in any medium, provided the original author and source are credited. 